\title{
Solvent Vapor Annealing, Defect Analysis, and Optimization of Self-assembly of Block Copolymers Using Machine Learning Approaches
}

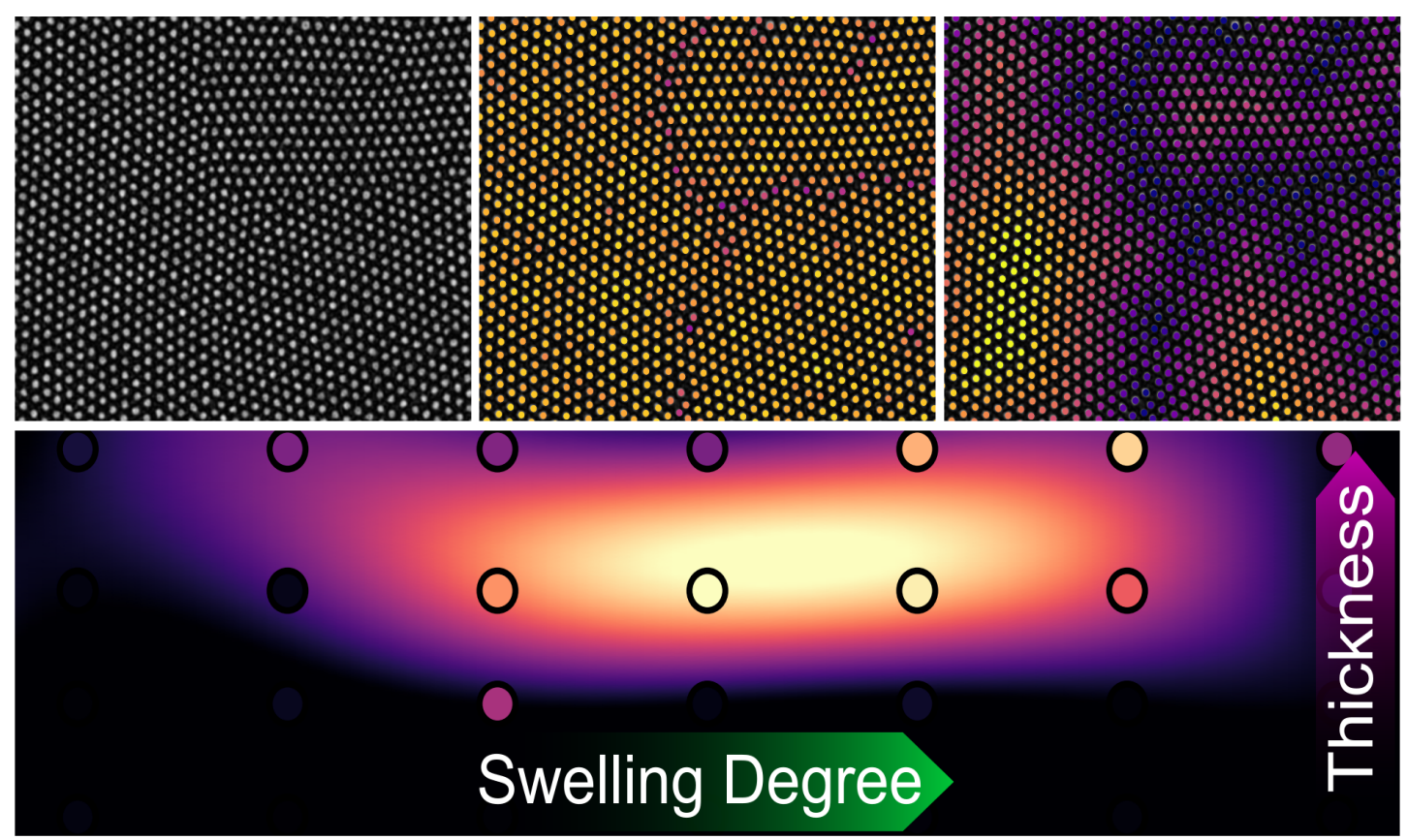

Gayashani Ginige, ${ }^{\mathrm{a}}$ Youngdong Song, ${ }^{\mathrm{b}}$ Brian C. Olsen, ${ }^{\mathrm{a}}$ Erik J. Luber, $*{ }^{, a}$ Cafer T. Yavuz, ${ }^{\mathrm{b}, \mathrm{c}, \mathrm{d}}$ Jillian M. Buriak, * a

${ }^{a}$ Department of Chemistry, University of Alberta, 11227-Saskatchewan Drive, Edmonton, AB T6G 2G2, Canada.

${ }^{b}$ Department of Chemical and Biomolecular Engineering, Korea Advanced Institute of Science and Technology (KAIST), 291 Daehak-ro, Yuseong-gu, Daejeon, 34141, Republic of Korea.

${ }^{\mathrm{C}}$ KAUST Catalysis Center (KCC), Physical Sciences and Engineering (PSE), King Abdullah University of Science and Technology (KAUST), Thuwal 23955-6900, Saudi Arabia

${ }^{\mathrm{d} A d v a n c e d ~ M e m b r a n e s ~ a n d ~ P o r o u s ~ M a t e r i a l s ~ C e n t e r ~(A M P M), ~ P h y s i c a l ~ S c i e n c e s ~ a n d ~}$ Engineering (PSE), King Abdullah University of Science and Technology (KAUST), Thuwal 23955-6900, Saudi Arabia

*E-mail: jburiak@ualberta.ca

*E-mail: eluber@ualberta.ca 


\begin{abstract}
Self-assembly of block copolymers (BCP) is an alternative patterning technique that promises sublithographic resolution and density multiplication. Defectivity of the resulting nanopatterns remains too high for many applications in microelectronics, and is exacerbated by small variations of processing parameters, such as film thickness, and fluctuations of solvent vapour pressure and temperature, among others. In this work, a solvent vapor annealing (SVA) flowcontrolled system is combined with Design of Experiments (DOE) and machine learning (ML) approaches. The SVA flow-controlled system enables precise optimization of the conditions of self-assembly of the high Flory-Huggins interaction parameter $(\chi)$ hexagonal dot array-forming BCP, PS- $b$-PDMS. The defects within the resulting patterns at various length scales are then characterized and quantified. The results show that defectivity of the resulting nanopatterned surfaces are highly dependent upon very small variations of the initial film thicknesses of the $\mathrm{BCP}$, as well as the degree of swelling under the SVA conditions. These parameters also significantly contribute to the quality of the resulting pattern with respect to grain coarsening, as well as the formation of different macroscale phases (single and double layers, and wetting layers). The results of qualitative and quantitative defect analyses are then compiled into a single figure of merit (FOM) using DOE and ML approaches, which enable identification of the narrow region of optimum conditions for SVA for a given BCP. The result of these analyses is a faster and less resource intensive route towards the production of low defectivity BCP dot arrays via rational determination of the ideal combination of processing factors. The DOE and machine learning-enabled approach is generalizable to scale-up of self-assembly-based nanopatterning for applications in electronics microfabrication.
\end{abstract}

\title{
Keywords:
}

Block copolymer; self-assembly; defect density; solvent vapor annealing; process control; directed self-assembly; memory; machine learning; high throughput 


\section{INTRODUCTION}

The spontaneous self-assembly of block copolymers (BCPs) to generate patterns and motifs with sub-lithographic resolution has been of great interest for over two decades as an alternative or complementary technique to photolithography. ${ }^{1-10}$ When integrated with sparse guiding morphological or chemical features produced via traditional lithography, these low cost polymer processing methods enable the generation of templates for production of sub-10 nm features with a high degree of long range order. ${ }^{11}$ This combination of self-assembly with lithography is termed directed self-assembly (DSA) and has been primarily directed towards applications in microelectronics, including memory storage materials, ${ }^{6,12,13}$ finFET, ${ }^{5,14,15}$ and vias. ${ }^{16-18}$ These nanopatterned substrates have also seen use as catalysts for growth of ordered nanowire arrays, ${ }^{19-}$ ${ }^{21}$ as a platform for protein detection, ${ }^{22,23}$ separation membranes, ${ }^{24-27}$ surface enhanced Raman spectroscopy (SERS) substrates, ${ }^{28-30}$ anti-reflective coatings in photovoltaics, ${ }^{31-33}$ and chemical and biomedical sensors. ${ }^{34-37}$

The self-assembly of a monolayer of a given BCP on a flat, featureless surface results in a polycrystalline morphology with uncorrelated nano- to micron-sized domains, with a significant concentration of defects. ${ }^{38}$ The ITRS (now IDRS roadmap) has specified a target of a maximum defectivity of $\sim 1$ per $100 \mathrm{~cm}^{-2},{ }^{39-43}$ which is several orders of magnitude lower than that quantified in patterns derived from these monolayers of self-assembled BCPs. Some kinetically trapped non-equilibrium metastable configurations in the free energy landscape persist even through long annealing times, which is an issue for high volume manufacturing on $300 \mathrm{~mm}$ wafers. ${ }^{11,18,42,44}$ In an attempt to avoid defect formation during the course of self-assembly, much effort has been directed towards the optimization of annealing techniques of the spin-coated BCP films, including solvent vapor, ${ }^{45-47}$ thermal (including microwave irradiation), ${ }^{48-51}$ a combination of solvent and thermal (solvothermal), ${ }^{52,53}$ laser annealing, ${ }^{54,55}$ shear flow, ${ }^{56,57}$ and annealing within an electric field. ${ }^{58}$ Lithography multiplication via DSA also reduces defect density as chemical and morphological features on the surface help to guide the BCP into the lowest energy equilibrium patterns. ${ }^{8,10,42,59,60}$ Defects still remain a challenge, however, particularly with smaller molecular weight BCPs with a high Flory-Huggins interaction parameter $\left(\chi_{N}>10.5\right)$ that produce the sub-20 $\mathrm{nm}$ periodicities, ${ }^{10,61,62}$ and features. ${ }^{9}$

To achieve the desired nanopattern via self-assembly, not only are the annealing parameters critical, but the initial thickness of the applied BCP monolayer must be precise, typically within one or two nanometers. ${ }^{11,63-68}$ The ideal thickness for a given BCP is unique, and 
depends upon the composition of the BCP and factors such as surface functionalization and energy, the use of a topcoat and other factors. ${ }^{8,10,42,62,64,69}$ Both experimental and computational results strongly link initial film thickness with the resulting self-assembled structure and persistent defects. ${ }^{18,70,71}$ Small fluctuations of thickness within a monolayer film can lead to different self-assembled structures as unfavorably thin domain thicknesses frustrate packing due to the buildup of strain. ${ }^{40,71}$ Periodicity of structures formed from self-assembled BCP nanopatterns may be dependent upon film thickness and other processing parameters, as has been recently shown with bottlebrush BCPs. ${ }^{63,72}$

The defects observed in self-assembled monolayers of BCPs that form hexagonal dot patterns are dominated by disclinations and dislocations, as well as point defects along grain boundaries between uncorrelated domains, multilayers, and the presence of lamellae. ${ }^{73-77}$ These hexagonal dot-based nanopatterns are of interest for memory materials and devices, applications that are more tolerant of defects than linear patterns for CMOS (e.g. vias), and thus the route towards commercialization appears more direct. "Further improvement of process-friendly techniques"1 is, however, still required, particularly over large areas. With the optimization of self-assembly of any new BCP, particularly high $\chi$ BCPs that may be challenging due to longlived metastable defects, or any change in processing conditions, annealing parameters will need to be re-evaluated. Empirical optimization of annealing of BCP films is time-consuming and if not rationally devised, will incompletely sample variable space. ${ }^{78,79}$ In an attempt to optimize these SVA parameters more efficiently with fewer laboratory resources (including time, one of the most precious resources), we use a full factorial experimental design complemented by machine learning (ML) approaches to identify the optimized SVA parameters. Prior to optimizing $\mathrm{BCP}$ dot arrays for minimum defectivity we first rigorously define what constitutes a defect, with respect to making bit-patterned media devices. Then, we define a figure of merit (FOM) that encompasses defect density and the spatial distribution of these defects at all relevant length scales. However, the factors involved in solvent annealing are non-orthogonal and correlated, with one alteration affecting other parameters of the film during self-assembly, and the effects playing out over different length scales. Given the difficulties of optimizing correlated systems with numerous input parameters, we show how ML techniques may be applied to characterize, quantify, predict, and ultimately minimize the defect densities of solvent-annealed hexagonal dotforming BCP nanopatterns. 


\section{EXPERIMENTAL METHODS}

\section{Materials}

Poly(styrene- $b$-dimethylsiloxane) (43k- $b$ - $8.5 \mathrm{k})$ diblock copolymer (PS- $b$-PDMS) with a polydispersity index of 1.04 was purchased from Polymer Source Inc. and used as-is. Toluene $(>99 \%)$ and tetrahydrofuran (THF, $>99 \%)$ were obtained from Fisher Scientific. Silicon wafers [ $<100>$, 4 in. diameter, thickness $525 \pm 25 \mathrm{~mm}$, p-type (boron doped), resistivity $<0.005 \mathrm{~W} . \mathrm{cm}]$ were purchased from WRS Materials. Sulfuric acid (96\%) and hydrogen peroxide (30\%) were purchased from Avantor Performance Materials.

\section{Silicon wafer dicing and cleaning}

Silicon wafers were diced into $1 \mathrm{~cm} \times 1 \mathrm{~cm}$ squares by a DSA 321 dicing saw and cleaned in fresh prepared piranha solution [3:1 v/v sulfuric acid (96\%)/hydrogen peroxide (30\%).

CAUTION: Piranha solution violently reacts with organic matter] for 15 minutes and washed with DI water and dried in a nitrogen stream.

\section{BCP thin-film self-assembly}

A bulk BCP solution with a concentration of $2 \mathrm{wt} \%$ in toluene was used to prepare solutions with concentrations ranging from $0.6 \mathrm{wt} \%$ to $1.4 \mathrm{wt} \%$. BCP thin films with thicknesses between 20 to $33 \mathrm{~nm}$ were prepared by spin casting $25 \mu \mathrm{l}$ of the desired polymer solution as shown in Figure S1 on piranha solution-cleaned Si chips at $8500 \mathrm{rpm}$ for $40 \mathrm{~s}$ with a WS WS-400BZ-6NPP/120 LITE spin-coater (Laurell Technologies Corporation). The initial film thickness was measured by ellipsometry and is the average of ellipsometric measurements taken at 5 different points on the chip. The variation of thickness with the concentration of the BCP solution is shown in the Figure S1.

\section{Controlled solvent vapor flow annealing system}

A complete procedure for how the annealing of a thin film of BCP on an oxide-capped Si chip using the controlled solvent vapor flow annealing system has been previously described. ${ }^{46}$ The initial film thickness (in $\mathrm{nm}$ ) and target swelling degree ( $\mathrm{SD}=$ swollen film thickness/initial film thickness) were entered into the program controlling the solvent annealing system. The dwell time was $300 \mathrm{~s}$, and ramp speed was $0.3 \mathrm{SD} / \mathrm{min}$, for all samples. It was ensured that the bubbler had enough solvent (THF) before commencing the annealing program, and 20 SCCM (standard cubic centimeter per minute) of Ar was bubbled into the solvent bubbler along with another pure Ar mixer to adjust the value automatically. The program automatically stopped solvent vapor 
flow at the end of the annealing, and the BCP film was deswelled by purging the annealing chamber with pure $\mathrm{Ar}^{46}$

\section{PS- $b$-PDMS thin film reactive ion etching (RIE) process}

All PS- $b$-PDMS thin films were etched with a Plasmalab $\mu$ Etch RIE using a two-step etch: The first step removed the surface wetting layer of PDMS on the BCP film by using a $\mathrm{CF}_{4}$ plasma $(100 \mathrm{sccm}), 100 \mathrm{mT}, 50 \mathrm{~W}$ for $8 \mathrm{~s}$. The second step, using an $\mathrm{O}_{2}$ plasma (100 sccm), $135 \mathrm{mT}, 30$ $\mathrm{W}$ for $60 \mathrm{~s}$ removed the PS and converted the PDMS block to $\mathrm{SiO}_{\mathrm{x}}$.

\section{Film characterization}

High magnification SEM micrographs were taken using a Hitachi S4800 scanning electron microscope $(15 \mathrm{kV}, 20 \mu \mathrm{A})$. Low resolution micrographs were taken using a Zeiss Sigma field emission scanning electron microscope $(15 \mathrm{kV}, 20 \mu \mathrm{A})$.

\section{Data processing}

SEM micrographs were processed to extract dot positions and areas of single layer phase using Gwyddion image analysis software. ${ }^{80}$ The background of the micrographs was removed using median scan line alignment followed by polynomial background removal and revolve arc for the single layer and dot micrographs respectively. A Gaussian filter was then used to reduce high frequency noise. The layer and dots were then marked by a brightness threshold found using Otzu's method. ${ }^{81}$ The ratio of single layer area was then averaged over 8 SEM micrographs for each set of conditions studied to get the final area percent of the single layer phase. The dot positions were used to compute the various metrics of the BCP dot arrays. The raw SEM micrographs for all SVA conditions, used to determine dot positions are shown in Figure S2.

The majority of the metrics used in this work are derived from the dot positions of the six nearest neighbors of each dot in relation to itself. The nearest neighbors are calculated using the ball tree algorithm in Scikit-learn. ${ }^{82}$ The dot pitch of each dot is calculated from the mean distance of its 6 nearest neighbors to itself. The cell orientation angle of each dot is the circular mean of the angle of each nearest neighbor vector modulus with $60^{\circ}$. This gives a cell orientation angle from -30 to $30^{\circ}$ for each dot. The registration error $\left(R_{e}\right)$ is calculated from the 6 nearest neighbors as explained in the supporting information. Defect distance is defined as the shortest distance from each dot to a defect. For the ML fit maps, the ensemble averages are taken as the mean of all non-defective dots.

ML fit maps were made to visualize the changes in the relevant metrics across all conditions tested. The fitting is performed using a radial basis function (RBF) kernel support 
vector machine (SVM) from Scikit-learn. ${ }^{82}$ After fitting, the function is remapped onto a grid to form an image for visualization.

\section{RESULTS AND DISCUSSION}

In this work we analyze the defects using ML approaches observed in different thicknesses of PS$b$-PDMS thin films that are solvent-annealed to a range of swelling degrees, plasma etched, and converted to silica nanopatterns, as outlined in Figure 1. This analysis of defects runs through a wide range of magnifications starting from defects in the lattice itself, to grains to different macro-scale phases in the film (wetting, single and double layers).

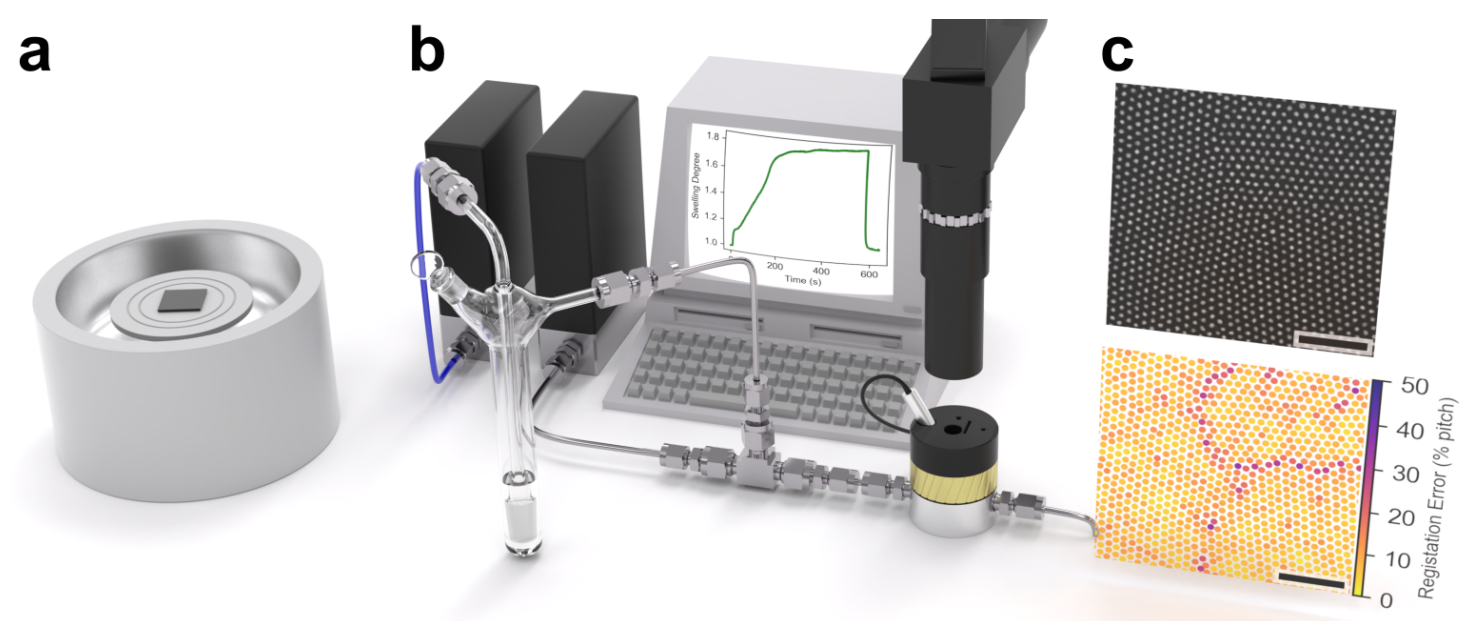

Figure 1. (a) The pre-annealed BCP film spin-coated onto silicon, locking in the initial film thickness. (b) Solvent vapor annealing (SVA) to a pre-determined swelling degree (SD) using a feedback-controlled annealing system. (c) Defect analysis of the resulting plasma-treated, silica nanopatterns imaged by SEM. Both scale bars are $250 \mathrm{~nm}$.

The focus of this work is the quantification, prediction, and minimization of defect density at all length scales of dot forming BCP films. However, other important metrics of the BCP dot array, such as pitch, can be easily determined and the effects of the solvent vapor annealing (SVA) parameter space on pitch analyzed. Shown in Figure 2 are dot maps of localized $\mathrm{BCP}$ dot pitch, where the pitch of each dot is determined by taking the mean distance of a dot and its six nearest neighbors. From both the low (Figure 2a) and high (Figure 2b) magnification maps we can see there is a clear trend of a small decrease in pitch with increasing swelling degree. These maps are color-coded, and thus the shift in color from yellow-green to blue-green is showing a decrease of pitch as the degree of swelling increases. As the degree of swelling is 
raised from 1.6 to 2.2, the average values of localized pitch range drop from $36.5 \mathrm{~nm}$ to from 32.5 $\mathrm{nm}$ (displayed graphically in Figure S3). Similar trends showing a decrease in pitch with increasing swelling degrees have been observed by others for different initial film thicknesses. ${ }^{45,83,84}$ At first glance it may seem somewhat counterintuitive that the pitch decreases as the swelling degree increases, however, it is important to realize that the film can only swell in the vertical direction perpendicular to the substrate, which causes the microdomains to be become non-spherical where they shrink in size in the in-plane direction and stretch in the out-of-plane direction. ${ }^{84}$ The decrease in pitch with increased swelling degree can be best understood by noting that the domain spacing, $L$, in the strong segregation limit is given by $L \sim N^{2 / 3} \chi_{\text {eff }}^{1 / 6} \cdot{ }^{85-88}$ Given that the effective Flory-Huggins interaction parameter $\chi$ decreases linearly with the amount of solvent incorporated into the film, ${ }^{84,89,90}$ the domain spacing is expected to decrease as swelling degree increases, as observed. Similarly, solvent molecules can fully incorporate into thinner films more easily compared to thicker films, leading to a decrease in pitch. ${ }^{91}$
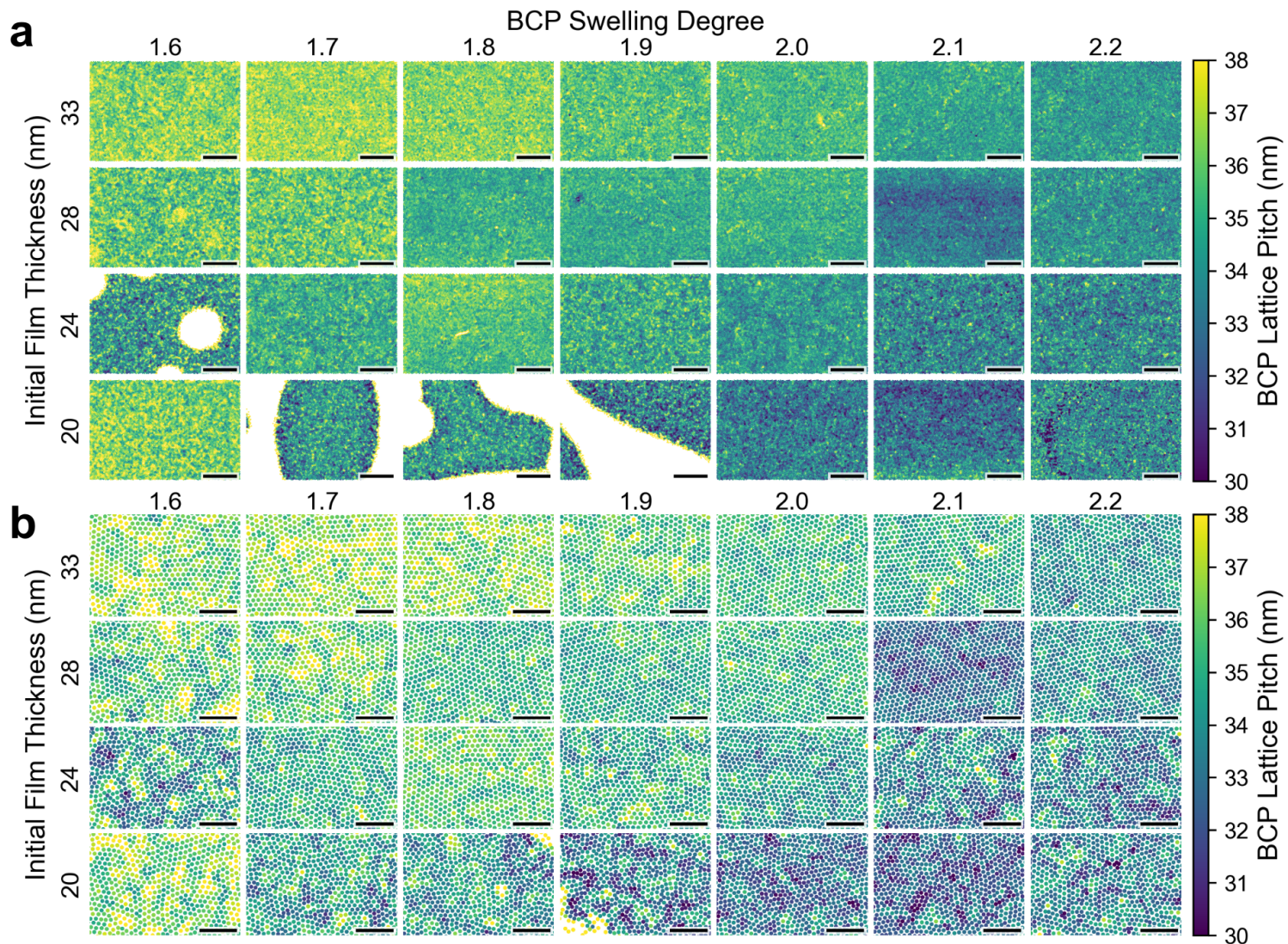

1.7 1.8

2.0

2.1
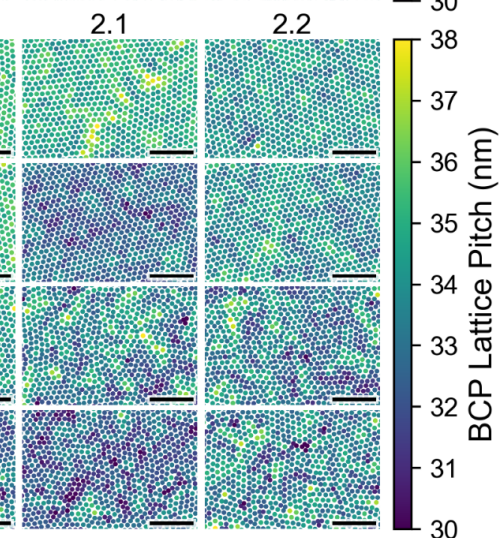

Figure 2. BCP lattice pitch as a function of SVA conditions. (a) Dot value maps showing dot locations for all 28 combinations of initial thickness of the PS- $b$-PDMS films and swelling degrees, indicated by the labelled rows and columns respectively, and showing color values for 
BCP lattice pitch in nm. All scale bars are $1 \mu \mathrm{m}$. (b) Details of (a) showing individual dots. All scale bars are $250 \mathrm{~nm}$.

Before quantitatively analyzing the defects in these BCP dot patterns, it is first useful to visualize the grains structures observed with different annealing conditions. The local cell orientation of each dot is mapped in Figure 3, which effectively maps out individual grains (regions where all the BCP dots have the same relative orientation). These results clearly demonstrate the large variability in long-range ordering as a function of initial film thickness and swelling degree. Some films comprise many very small, sub-100 nm grains, and others are uniform over micron scales. At a film thickness of $20.0 \mathrm{~nm}$, the annealed films have extremely small grains (on the order of a few lattice pitches) and the grains themselves have a low degree of local orientational ordering (vide infra). There also appears to be regions of optimal grain size and local orientational ordering. For example, at an initial film thickness of $24.0 \mathrm{~nm}$, the grain size increases as the swelling degree increases, reaching a maximum size, but then decreasing as the swelling degree is further increased; the local ordering also seems to significantly decrease. The initial increase in grain size with increased swelling degree is a result of the intended action of solvent annealing, where increased solvent vapour incorporated into the film increases chain mobility, allowing for the formation of ordered lattice structures with lower configurational energy. ${ }^{45}$ However, this increase in chain mobility is always accompanied by a decrease in segregation strength, and thus as the segregation strength approaches the order-disorder transition (ODT) the local microdomain ordering begins to decrease. ${ }^{45,92}$ These competing processes of chain mobility and segregation strength explain the trends observed in Figure 3 where there will be an optimal swelling degree for a given initial film thickness to maximize grain size and longrange ordering. 


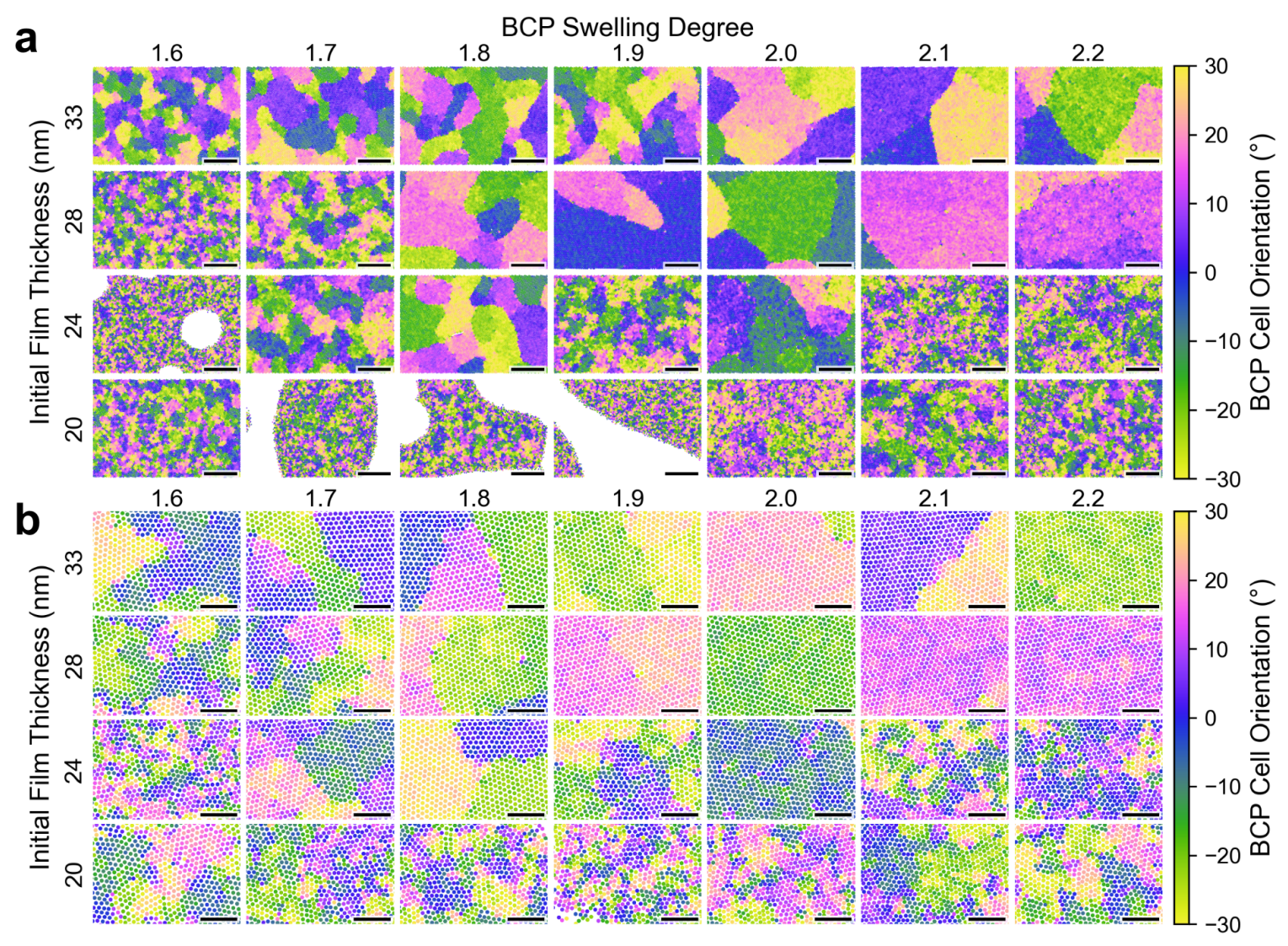

Figure 3. BCP lattice cell orientation as a function of SVA conditions. (a) Dot value maps showing dot locations for all 28 combinations of initial thickness of the PS- $b$-PDMS films, and swelling degrees, indicated by the labelled rows and columns respectively, and showing color values for BCP lattice cell orientation in degrees. All scale bars are $1 \mu \mathrm{m}$. (b) Details of (a) showing individual dots. All scale bars are $250 \mathrm{~nm}$.

Although these BCP cell orientation maps provide a useful qualitative sense of grain size and long-range ordering, it is necessary to quantitatively define and measure defects in order to apply ML methods to optimize the SVA processing. One of the most conventional methods for identifying defects is to determine the coordination number, where any non-6 fold coordinated dots are marked as defects. ${ }^{93}$ Maps of the local coordination number are shown in Figure S4, which very clearly identify topological point defects like dislocations and disclinations having 5and 7-fold coordinated sites. Although the coordination number very easily identifies point defects, it does not encode for fluctuations and deviations from hexagonal ideality, which is a significant concern in these soft BCP lattices during SVA. These deviations in local hexagonality are important to consider when attempting to quantify the defectivity of these $\mathrm{BCP}$ lattices and their broader applications in bit-patterned media. Specifically, it is easy to imagine a lattice with only 6 -fold coordinated sites, but have sufficiently large local fluctuations in dot positioning to 
cause read/write errors. With this concept in mind, we define the metric of registration error $\left(R_{e}\right)$ in order to identify fluctuational defects.

Simply put, the $R_{e}$ of a given dot is a measure of the average distance of its six nearest neighbours from the corresponding perfect hexagonal coordination shell. Expressed mathematically, the $R_{e}$ of any dot is given by

$$
R_{e}=\left(\frac{1}{6 L_{0}} \sqrt{\sum_{n=1}^{6}\left(x_{n}^{\prime}-x_{n}^{\mathrm{NN}}\right)^{2}+\left(y_{n}^{\prime}-y_{n}^{\mathrm{NN}}\right)^{2}}\right) \times 100 \%
$$

where $L_{0}$ is the median pitch of the entire BCP dot array, $x_{n}^{\mathrm{NN}}$ ) and $y_{n}^{\mathrm{NN}}$ ) are the $x, y$ coordinates of the six nearest neighbours (sorted in a clockwise fashion) and $x_{n}^{\prime}$ and $y_{n}^{\prime}$ are the coordinates of the regular hexagonal (with a pitch equal to $L_{0}$ ) that minimizes the value of $R_{e}$. Further detail of how $R_{e}$ is calculated is given in the Supporting Information. 


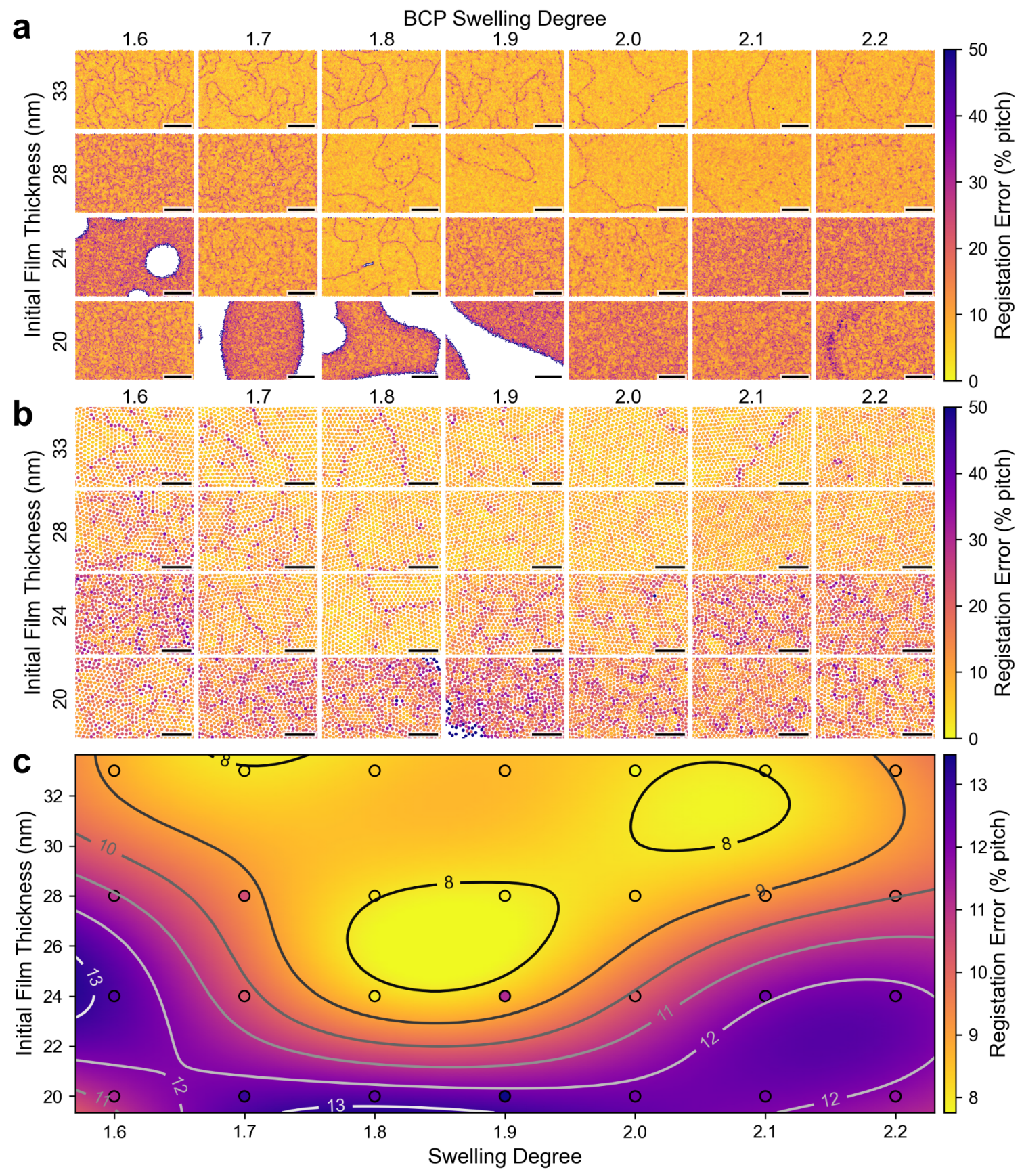

Figure 4. Registration error as a function of SVA conditions. (a) Dot value maps showing dot locations for all 28 combinations of initial thickness of the PS- $b$-PDMS films, and swelling degrees, indicated by the labelled rows and columns respectively, and showing color values for registration error in percent dot pitch. All scale bars are $1 \mu \mathrm{m}$. (b) Details of (a) showing individual dots. All scale bars are $250 \mathrm{~nm}$. (c) Support vector machine fitting using a radial basis function kernel of the mean registration error where dots with a registration error greater than $17 \%$ have been excluded as defects. 
This effect of increased lattice deviations from perfect hexagonality is illustrated in Figure 4 for an initial film thickness of $28.0 \mathrm{~nm}$. At a low swelling degree of 1.6, the lattice has a very large number of point defects, but the number of defects sharply decreases as the swelling degree is increased to 1.8 , at which point almost all of the point defects belong to the grain boundaries. However, as the swelling degree is further increased, a very subtle but important change can be observed in the $R_{e}$ maps, where the map appears to be darker purple (higher registration error) despite a similar number of point defects/grain boundaries. This increase of registration error is due to the average value of $R_{e}$ increasing due to increased fluctuations in the local hexagonal coordination shell of $\mathrm{BCP}$ dots. This increase in the average $R_{e}$ for 6-fold coordinated sites is believed to be a result of the segregation strength decreasing as the swelling degree increases. The relationship between the changes in the global average of $R_{e}$ and SVA conditions is shown in the Figure 4c, which is computed by ML fitting of the measured $R_{e}$ values. This map clearly identifies an optimal zone of SVA parameters that minimizes the global average of $R_{e}$.

Using this measure of registration error, we can define what constitutes a fluctuational defect in a physically meaningful way, as it relates to reading/writing in bit patterned media. Traditional measures of hexagonal lattice fluctuations, such as the local orientational order parameter, $\psi_{6},{ }^{94}$ are also very sensitive to fluctuations in hexagonal ordering, where mappings of $\psi_{6}$ are shown in Figure S5 and are very similar in appearance to the $R_{e}$ mappings in Figure 4. However, there is no good way to relate values of $\psi_{6}$ to the possibility of a fluctuational defect, outside of choosing an arbitrary cut-off. Conversely, since $R_{e}$ is a measure of actual distance, we can define a fluctuational defect when $R_{e}>r_{0} / L_{0} \times 100$, where $r_{0}$ is the effective diameter of the BCP dots. From SEM data we estimate the average dot size to be $\sim 8 \mathrm{~nm}$ (see Figure S6), which is an overestimation due to charging effects and non-zero spot size of the electron beam. To more accurately estimate the true/effective size of the dots, we assume that they are at least 2 $\mathrm{nm}$ smaller than measured by SEM, giving an effective dot radius of $\sim 6 \mathrm{~nm}$. Therefore, all dots with a $R_{e}>17$ are defined as a fluctuational (or point) defect. The physical interpretation of this defect definition is clear: if the read/write head is moved to the expected location of a nearest neighbour (based on perfect hexagonal lattice) and the registration error is greater than $17 \%$, the dot will not be detected.

Having defined defects as above (counting both point and fluctuational defects) we would like to compute an appropriate figure of merit (FOM) for a given BCP dot array, which measures how desirable that BCP array would be if used for bit patterned media. Although a raw 
average of the defect density is informative and simple to measure, it does not tell us how the defects are distributed in space. For instance, are they all located on grain boundaries or are they uniformly distributed throughout? As such, we define the metric of local defect distance, which is simply the minimum distance between a BCP dot and a defect (normalized by BCP pitch). Shown in Figure 5 are the local defect distance maps for each SVA condition tested. It is worth noting that these local defect distance maps are simply the Euclidean distance transform ${ }^{95}$ of the defect maps (Figure S7). In contrast to the $R_{e}$ maps, the local defect distance maps show that, from patterned bit media perspective, better BCP arrays can be made from films with initial thicknesses of $28 \mathrm{~nm}$, compared to $33 \mathrm{~nm}$ (i.e. there are much larger regions that do not contain any defects).

a
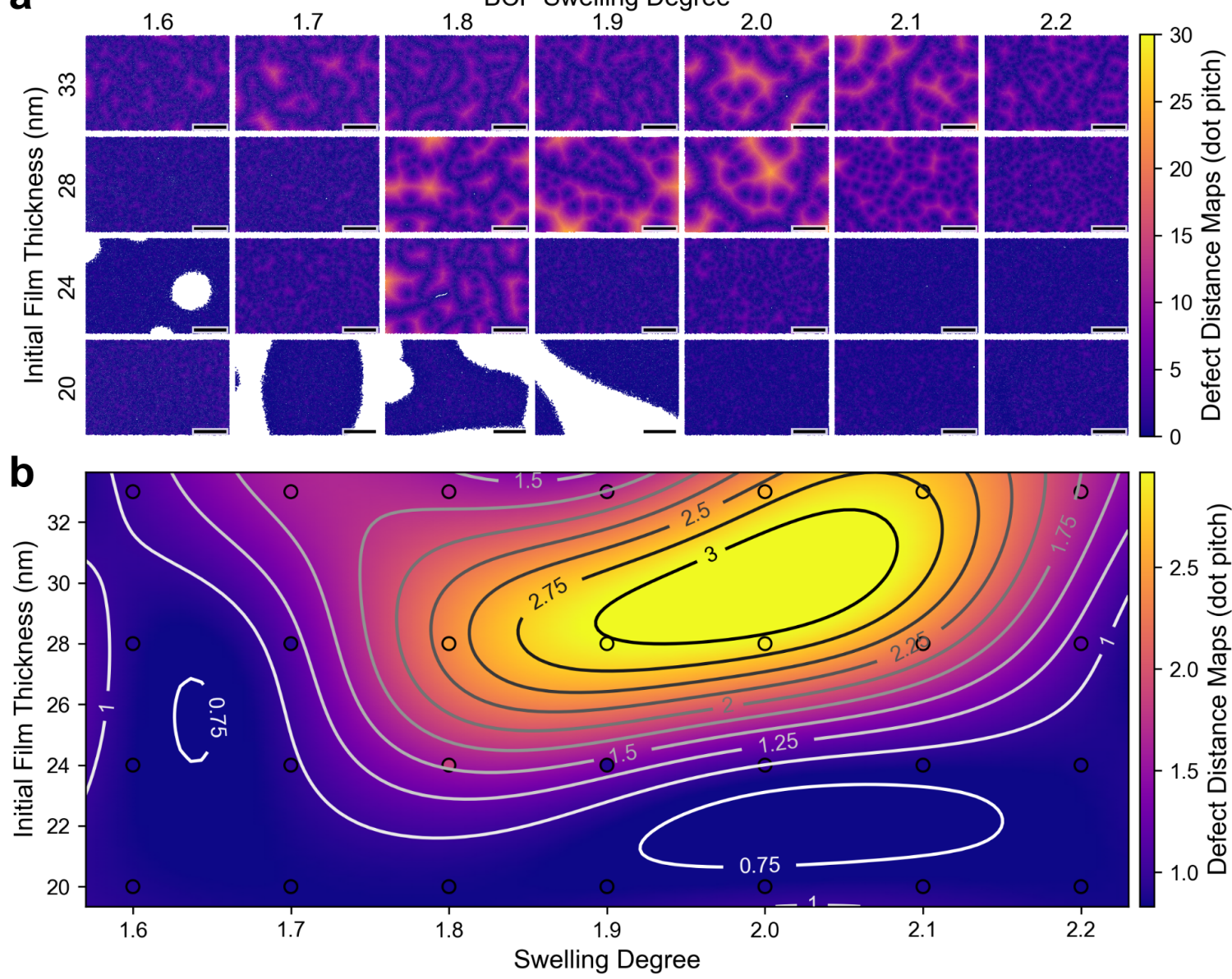

Figure 5. Defect distance as a function of SVA conditions. (a) Dot maps where the color value is the distance to the nearest defect. A defect is defined as having a registration error less than $17 \%$ where all of the vacancies and grain boundaries are included. All scale bars are $1 \mu \mathrm{m}$. (b) Support vector machine fitting using a radial basis function kernel of the effective defect distance per SVA condition. This effective defect distance represents a device size (measured in lattice pitch) that would have an $80 \%$ yield of devices with no defects. 
This metric of defect distance can be directly related to yield and size of bit-patterned media devices, where an average of the local defect distance of the entire BCP array gives a measure of how large a device could be fabricated without any defects. Specifically, the effective defect distance is defined as the $20 \%$ percentile of local defect distance for a given BCP array (this distance would equal the device size that would have an $\sim 80 \%$ yield, meaning no defects for $80 \%$ of devices). A map of the effective defect distance (as a function of SVA conditions) is shown in Figure 5b, which clearly identifies a narrow region of optimal annealing conditions centered around an initial thickness of $\sim 30 \mathrm{~nm}$ and swelling degree of $\sim 2.0$.

The phase behavior of dot-forming BCP thin films (such as PS- $b$-PDMS used in this work) is quite complex, as lamellae/wetting layers, single layer hexagonal or multiple stacked layers of hexagonal dots can be formed. Which phases form in a BCP thin film is strongly dependent on initial thickness, which needs to be commensurate with the native BCP pitch. ${ }^{65,66,96-}$ ${ }^{99}$ Moreover, during SVA the film swells in thickness, often resulting in the formation of multiple layers. ${ }^{76,77}$ The final areal fraction of single layers (or desired phase) over the entire substrate is one of the most important (and often unreported) metrics of BCP thin film annealing. From the perspective of bit-patterned media devices, any regions that are not the desired phase would effectively consist of $100 \%$ defects. 
a

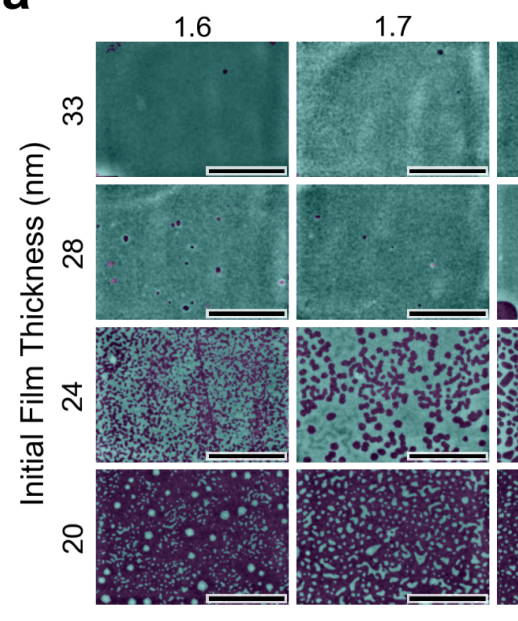

BCP Swelling Degree
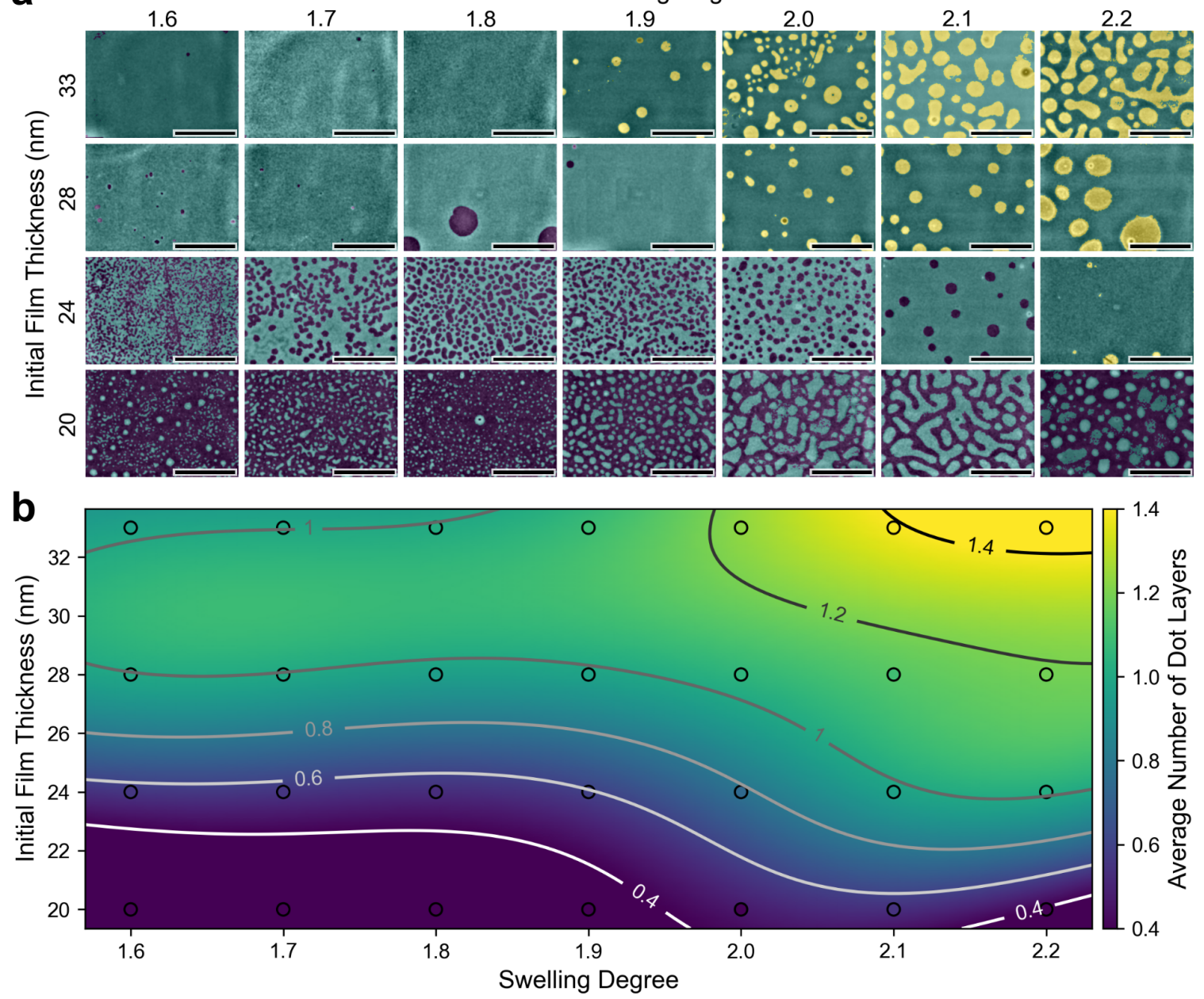

Figure 6. Number of hexagonal dot layers as a function of SVA conditions. (a) False color low magnification SEM micrographs showing wetting layers in dark blue, single layers in green and double layers in yellow. All scale bars are $50 \mu \mathrm{m}$. (b) Support vector machine fitting using a radial basis function kernel of the area averaged number of dot layers, where "0" represents a wetting layer, " 1 " represents a single layer of dots and "2" represents double layer areas. Scale bar is set to $0.4-1.4$ to improve contrast.

Shown in Figure 6a are false colored low magnification SEM images $(100 \mu \mathrm{m} \times 100$ $\mu \mathrm{m})$, where purple shows lamellae/wetting layers, green is a single layer and yellow is a double layer. The first thing to note is that under almost all SVA conditions there are generally two phases present, either a combination of lamellae/wetting and single layer phases, or single layer and double layer phases. To better understand this phase behaviour, the average number of layers (per SVA condition) is measured as

$$
N_{\text {ave }}=0 \times f_{l}+1 \times f_{s}+2 \times f_{d}
$$

where $f_{l}$ is the area fraction of lamellae, $f_{s}$ is the fraction of single layers and $f_{d}$ is the fraction of double layers. Shown in Figure $6 \mathrm{~b}$ is a ML fit to the average number of layers, as a function of 
SVA conditions, which reveals several important features of the phase formation. As expected, we see that the average number of layers generally increases as the initial thickness and swelling degree increases. However, at swelling degrees less than $\sim 1.8$, the average number of layers is essentially insensitive to changes in the swelling degree, which is likely due to the very slow kinetics at these swelling degrees. One of the most useful features of this map is identifying the SVA conditions where the average layer number is close to 1.0 and is insensitive to fluctuations in SVA conditions. Specifically, from a practical device manufacturing perspective, there will always be non-uniformities in film thickness, mass transport, and solvent uptake over the entire substrate. ${ }^{49}$ As such, it is important to consider these system sensitivities to inputs when selecting SVA conditions, where regions of a plateau with an average layer number of 1.0 are most desirable.

Making use of all of the above analysis, we can define a single FOM that can be used to identify the optimal SVA conditions for producing BCP dot arrays for bit-patterned media. The FOM we choose is simply the defect distance, modified by the fraction of single layers, as we need to proportionally reduce the effective defect distance to account for the fact that non-single layer regions have a $100 \%$ defect density. A map of this FOM is shown in Figure 7c, which is the product of the defect distance (Figure 7a) and fraction of single layer (Figure 7b). It is noted that the optimal FOM does not correspond to either the optimal defect distance or single layer fraction regions, but is rather the intersection of these two regions. This conclusion highlights the tradeoff between single layer fraction and defect distance: at lower swelling degrees there is a large region of near $100 \%$ single layer, but the defectivity is much worse compared to that observed at higher swelling degrees. At higher swelling degrees, however, the decrease in defectivity is offset by a reduction in the number of single layers. This leads to a relatively narrow region of SVA conditions with the optimal FOM, which is located between a swelling degree of 1.9 to 2.0 and an initial film thickness of 28 to $30 \mathrm{~nm}$. These conditions are clearly specific to this BCP with this molecular weight, but the methods presented here are completely general and can be utilized for different BCPs. 

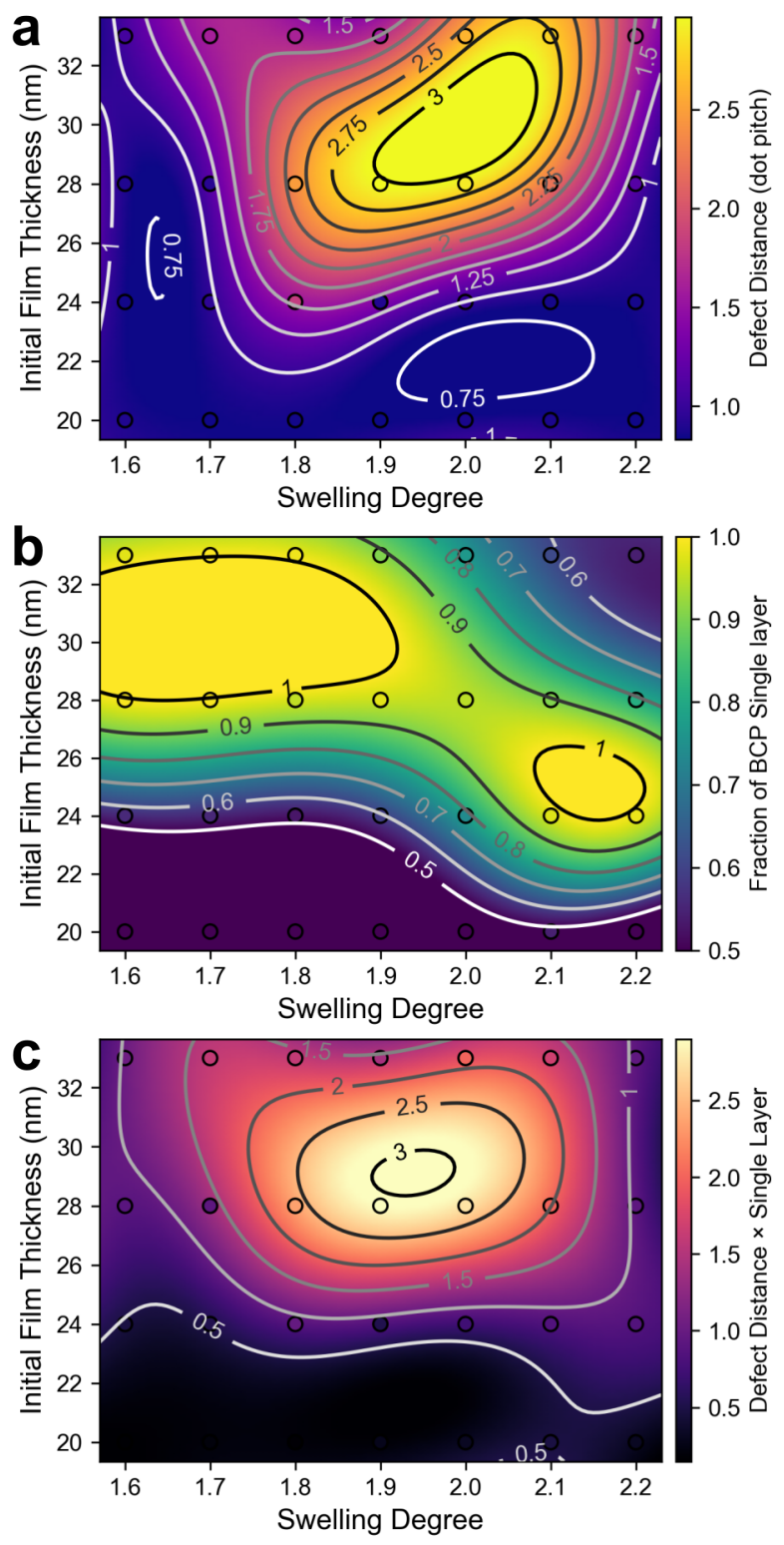

Figure 7. Support vector machine fitting using a radial basis function kernel of the (a) Defect distance of the $20 \%$ percentile (Where a defect is defined as having a registration error above $17 \%$ where majority of the vacancies and grain boundaries are included. This value would represent a device size in dot pitch that would have a $80 \%$ yield(no defects)), (b) Fraction single layer and (c) Multiplication of fraction single layer by defect distance.

Although not presented in this work, several further refinements/modifications of this method can be applied for SVA optimization of nanopatterns derived from BCP films. As presented in our previous work on optimization of thin film organic photovoltaic cells, ${ }^{78}$ the coupling of Design of Experiments with Machine Learning, done in an iterative manner can be a very powerful tool for optimization. Specifically, in this work, we have carried out what would be considered a full factorial design, where the parameter space is sampled uniformly on a dense 
grid. The number of experiments could be reduced by applying a generalized subset design, ${ }^{100}$ and still perform the ML fitting to identify regions of interest with a high FOM. These regions could then be further sampled using DOE+ML in an iterative fashion. Furthermore, once the first round optimization is done to identify these promising regions, even greater FOMs could be achieved if the annealing time was investigated in these regions, as longer annealing times will likely reduce defectivity in the films.

\section{SUMMARY}

Application of block copolymer self-assembly for pattern generation with sublithographic resolution requires minimization of defect densities at both the nano- and macroscales. Controlling and optimizing a large number of correlated and convoluted process parameters during processing, and subsequent data analysis is time consuming if executed in an empirical fashion, and may not arrive at the optimum conditions. In this work, we investigated the influence of the small thickness variations and swelling degrees on the final morphology of the BCP patterns and how to optimize these SVA parameters for fabrication of devices. A full factorial experimental design was used to probe the SVA parameter space, and a figure of merit was formulated to evaluate the quality of the resultant BCP nanopatterns, accounting for multiple length scales and the requirements of bit patterned media. Machine learning was then utilized to fit the SVA parameter space and identify the optimal annealing conditions with respect to the figure of merit. This approach is generalizable to optimize, analyze, and arrive at predictions of defect densities for different combinations of materials and processes for device fabrication. This combination of techniques may be applied to any combination of materials and processes to optimize, analyse, predict, and minimize the defect densities in any given pattern.

\section{ASSOCIATED CONTENT}

\section{Supporting Information}

Plots showing relationship between initial film thickness and concentration of $\mathrm{BCP}$ solution, SEM micrographs of BCP dot patterns, low magnification SEM micrographs colored by BCP phase, ML maps and/or dot maps as function of swelling degree and initial film thickness of: $\mathrm{BCP}$ pitch, coordination number, orientational order parameter, fluctuational defects; histograms of: $\mathrm{BCP}$ dot size, registration error, $\mathrm{BCP}$ cell orientation, $\mathrm{BCP}$ pitch, defect distance.

\section{Financial Interests}

The authors declare no competing financial interests. 


\section{AUTHOR INFORMATION}

\section{Corresponding Authors}

Erik J. Luber - Department of Chemistry, University of Alberta, Edmonton, AB T6G 2G2,

Canada; ORCID: 0000-0003-1623-0102;

Email: eluber@ualberta.ca

Jillian M. Buriak - Department of Chemistry, University of Alberta, Edmonton, AB T6G 2G2,

Canada; ORCID: 0000-0002-9567-4328;

Email: jburiak@ualberta.ca

\section{Authors}

Gayashani Ginge - Department of Chemistry, University of Alberta, Edmonton, AB T6G 2G2, Canada; ORCID: 0000-0002-4434-9648;

Youngdong Song - Department of Chemical and Biomolecular Engineering, Korea Advanced Institute of Science and Technology (KAIST), 291 Daehak-ro, Yuseong-gu, Daejeon, 34141, Republic of Korea; ORCID: 0000-0003-2565-2091

Cafer T. Yavuz - KAUST Catalysis Center (KCC), Physical Sciences and Engineering (PSE), King Abdullah University of Science and Technology (KAUST), Thuwal 23955-6900, Saudi Arabia; ORCID: 0000-0003-0580-3331

Brian C. Olsen - Department of Chemistry, University of Alberta, Edmonton, AB T6G 2G2, Canada; ORCID: 0000-0001-9758-3641

\section{ACKNOWLEDGMENTS}

This work was supported by NSERC (grant number RGPIN-2018-04294), Alberta Innovates Technology Futures (grant number AITF iCORE IC50-T1 G2013000198), the Canada Research Chairs program (CRC 207142), and the International Research \& Development Program of the National Research Foundation of Korea (NRF) funded by the Ministry of Science and ICT (grant number 2017K1A3A1A12029286). The University of Alberta Centre for Nanofabrication (the nanoFAB) and the National Research Council-Edmonton are thanked for the use of facilities. 


\section{REFERENCES}

(1) Chen, Y.; Xiong, S. Directed Self-Assembly of Block Copolymers for Sub-10 nm Fabrication. Int. J. Extrem. Manuf. 2020, 2, 032006.

(2) Ait-Ferhat, D.; Juliard, V.; Stauffer, G.; Torres, J. A. Combining Lithography and Directed Self Assembly for the Manufacturing of Vias: Connections to Graph Coloring Problems, Integer Programming Formulations, and Numerical Experiments. European Journal of Operational Research 2020, 280, 453-468.

(3) Wan, L.; Ruiz, R. Path to Move Beyond the Resolution Limit with Directed SelfAssembly. ACS Appl. Mater. Interfaces 2019, 11, 20333-20340.

(4) Cummins, C.; Pino, G.; Mantione, D.; Fleury, G. Engineering Block Copolymer Materials for Patterning Ultra-Low Dimensions. Mol. Syst. Des. Eng. 2020, 5, 1642-1657.

(5) Liu, C.-C.; Franke, E.; Mignot, Y.; Xie, R.; Yeung, C. W.; Zhang, J.; Chi, C.; Zhang, C.; Farrell, R.; Lai, K.; Tsai, H.; Felix, N.; Corliss, D. Directed Self-Assembly of Block Copolymers for 7 Nanometre FinFET Technology and Beyond. Nat. Electron. 2018, 1, 562-569.

(6) Liu, C.-C.; Mignot, Y.; Chi, C.; Farrell, R.; Lai, K.; Guo, J.; Sha, J.; Glodde, M.; Muramatsu, M.; Ido, Y.; Felix, N.; Hetzer, D.; Metz, A.; Corliss, D. The Integration of 193i and DSA for BEOL Metal Cuts/Blocks Targeting Sub-20 nm Tip-to-Tip CD. In Novel Patterning Technologies 2018; International Society for Optics and Photonics, 2018; Vol. 10584, p $105840 \mathrm{~L}$.

(7) Kihara, N.; Yamamoto, R.; Sasao, N.; Shimada, T.; Yuzawa, A.; Okino, T.; Ootera, Y.; Kamata, Y.; Kikitsu, A. Fabrication of 5 Tdot/in. ${ }^{2}$ Bit Patterned Media with Servo Pattern Using Directed Self-Assembly. Journal of Vacuum Science \& Technology B 2012, 30, 06FH02.

(8) Stein, A.; Wright, G.; Yager, K. G.; Doerk, G. S.; Black, C. T. Selective Directed SelfAssembly of Coexisting Morphologies Using Block Copolymer Blends. Nat. Commun. 2016, 7 , 12366.

(9) Arias-Zapata, J.; Böhme, S.; Garnier, J.; Girardot, C.; Legrain, A.; Zelsmann, M.

Ultrafast Assembly of PS-PDMS Block Copolymers on $300 \mathrm{~mm}$ Wafers by Blending with Plasticizers. Adv. Funct. Mater. 2016, 26, 5690-5700. 
(10) Kim, J. Y.; Liu, P.; Maher, M. J.; Callan, D. H.; Bates, C. M.; Carlson, M. C.; Asano, Y.; Blachut, G.; Rettner, C. T.; Cheng, J. Y.; Sunday, D. F.; Kline, R. J.; Sanders, D. P.; Lynd, N. A.; Ellison, C. J.; Willson, C. G.; Baiz, C. R. Spatial Control of the Self-Assembled Block Copolymer Domain Orientation and Alignment on Photopatterned Surfaces. ACS Appl. Mater. Interfaces 2020, 12, 23399-23409.

$$
\text { Li, W.; Müller, M. Directed Self-Assembly of Block Copolymers by Chemical or }
$$

Topographical Guiding Patterns: Optimizing Molecular Architecture, Thin-Film Properties, and Kinetics. Progress in Polymer Science 2016, 54-55, 47-75.

(12) Tu, K.-H.; Fernandez, E.; Almasi, H.; Wang, W.; Otero, D. N.; Ntetsikas, K.; Moschovas, D.; Avgeropoulos, A.; Ross, C. A. Magnetic Reversal and Thermal Stability of CoFeB Perpendicular Magnetic Tunnel Junction Arrays Patterned by Block Copolymer Lithography. Nanotechnology 2018, 29, 275302.

(13) Frascaroli, J.; Brivio, S.; Ferrarese Lupi, F.; Seguini, G.; Boarino, L.; Perego, M.; Spiga, S. Resistive Switching in High-Density Nanodevices Fabricated by Block Copolymer SelfAssembly. ACS Nano 2015, 9, 2518-2529.

(14) Mitra, J.; Torres, A.; Pan, D. Z. Process, Design Rule, and Layout Co-Optimization for DSA Based Patterning of Sub-10 nm Finfet Devices. In Emerging Patterning Technologies; International Society for Optics and Photonics, 2017; Vol. 10144, p 101440G.

(15) Li, D.; Chien, C.; Wei, X.; Huang, Y.; Qu, X.; Chang, T.; Xiong, S. Sub-10 nm Silicon FinFET Devices on SOI Substrate Made by Block Copolymer Lithography. In 2018 14th IEEE International Conference on Solid-State and Integrated Circuit Technology (ICSICT); 2018; pp $1-3$.

(16) Doise, J.; Bekaert, J.; Chan, B. T.; Hori, M.; Gronheid, R. Via Patterning in the 7-nm Node Using Immersion Lithography and Graphoepitaxy Directed Self-Assembly. JM3 2017, 16, 023506 .

(17) Murugesan, M.; Fukushima, T.; Bea, J. C.; Hashimoto, H.; Koyanagi, M. Intra- and InterChip Electrical Interconnection Formed by Directed Self Assembly of Nanocomposite Containing Diblock Copolymer and Nanometal. In 2018 IEEE International Reliability Physics Symposium (IRPS); 2018; pp 4D.2-1-4D.2-7. 
(18) Rottler, J.; Müller, M. Kinetic Pathways of Block Copolymer Directed Self-Assembly: Insights from Efficient Continuum Modeling. ACS Nano 2020, 14, 13986-13994.

(19) Huang, Y.; Kim, T. W.; Xiong, S.; Mawst, L. J.; Kuech, T. F.; Nealey, P. F.; Dai, Y.; Wang, Z.; Guo, W.; Forbes, D.; Hubbard, S. M.; Nesnidal, M. InAs Nanowires Grown by MetalOrganic Vapor-Phase Epitaxy (MOVPE) Employing PS/PMMA Diblock Copolymer Nanopatterning. Nano Lett. 2013, 13, 5979-5984.

(20) Kim, J. Y.; Lim, J.; Jin, H. M.; Kim, B. H.; Jeong, S.-J.; Choi, D. S.; Li, D. J.; Kim, S. O. 3D Tailored Crumpling of Block-Copolymer Lithography on Chemically Modified Graphene. Adv. Mater. 2016, 28, 1591-1596.

(21) Wei, W.; Samad, L.; Choi, J. W.; Joo, Y.; Way, A.; Arnold, M. S.; Jin, S.; Gopalan, P. Synthesis of Molybdenum Disulfide Nanowire Arrays Using a Block Copolymer Template. Chem. Mater. 2016, 28, 4017-4023.

(22) Stel, B.; Gunkel, I.; Gu, X.; Russell, T. P.; De Yoreo, J. J.; Lingenfelder, M. Contrasting Chemistry of Block Copolymer Films Controls the Dynamics of Protein Self-Assembly at the Nanoscale. ACS Nano 2019, 13, 4018-4027.

(23) Xie, T.; Chattoraj, J.; Mulcahey, P. J.; Kelleher, N. P.; Gado, E. D.; Hahm, J. Revealing the Principal Attributes of Protein Adsorption on Block Copolymer Surfaces with Direct Experimental Evidence at the Single Protein Level. Nanoscale 2018, 10, 9063-9076.

(24) Zhu, G.; Ying, Y.; Li, X.; Liu, Y.; Yang, C.; Yi, Z.; Gao, C. Isoporous Membranes with Sub-10 nm Pores Prepared from Supramolecular Interaction Facilitated Block Copolymer Assembly and Application for Protein Separation. Journal of Membrane Science 2018, 566, 2534.

(25) Lee, D.-S.; Park, S.; Han, Y. D.; Lee, J. E.; Jeong, H. Y.; Yoon, H. C.; Jung, M. Y.; Kim, S. O.; Choi, S.-Y. Selective Protein Transport Through Ultra-Thin Suspended Reduced Graphene Oxide Nanopores. Nanoscale 2017, 9, 13457-13464.

(26) Zhou, C.; Segal-Peretz, T.; Oruc, M. E.; Suh, H. S.; Wu, G.; Nealey, P. F. Fabrication of Nanoporous Alumina Ultrafiltration Membrane with Tunable Pore Size Using Block Copolymer Templates. Adv. Funct. Mater. 2017, 27, 1701756. 
(27) Bertrand, A.; Bousquet, A.; Lartigau-Dagron, C.; Billon, L. Hierarchically Porous BioInspired Films Prepared by Combining "Breath Figure" Templating and Selectively Degradable Block Copolymer Directed Self-Assembly. Chem. Commun. 2016, 52, 9562-9565.

(28) Jin, H. M.; Kim, J. Y.; Heo, M.; Jeong, S.-J.; Kim, B. H.; Cha, S. K.; Han, K. H.; Kim, J. H.; Yang, G. G.; Shin, J.; Kim, S. O. Ultralarge Area Sub-10 nm Plasmonic Nanogap Array by Block Copolymer Self-Assembly for Reliable High-Sensitivity SERS. ACS Appl. Mater. Interfaces 2018, 10, 44660-44667.

(29) Lin, C.-W.; Chang, S.-H.; Huang, C.-C.; Lin, C.-H. Plasmonic Nanocavities Fabricated by Directed Self-Assembly Lithography and Nanotransfer Printing and Used as SurfaceEnhanced Raman Scattering Substrates. Microelectronic Engineering 2020, 227, 111309.

(30) Akinoglu, G. E.; Mir, S. H.; Gatensby, R.; Rydzek, G.; Mokarian-Tabari, P. Block Copolymer Derived Vertically Coupled Plasmonic Arrays for Surface-Enhanced Raman Spectroscopy. ACS Appl. Mater. Interfaces 2020, 12, 23410-23416.

(31) Adak, D.; Ghosh, S.; Chakraborty, P.; Srivatsa, K. M. K.; Mondal, A.; Saha, H.; Mukherjee, R.; Bhattacharyya, R. Non Lithographic Block Copolymer Directed Self-Assembled and Plasma Treated Self-Cleaning Transparent Coating for Photovoltaic Modules and Other Solar Energy Devices. Sol. Energy Mater. Sol. Cells 2018, 188, 127-139.

(32) Rahman, A.; Ashraf, A.; Xin, H.; Tong, X.; Sutter, P.; Eisaman, M. D.; Black, C. T. Sub50-Nm Self-Assembled Nanotextures for Enhanced Broadband Antireflection in Silicon Solar Cells. Nat. Commun. 2015, 6, 5963.

(33) Mokarian-Tabari, P.; Senthamaraikannan, R.; Glynn, C.; Collins, T. W.; Cummins, C.; Nugent, D.; O’Dwyer, C.; Morris, M. A. Large Block Copolymer Self-Assembly for Fabrication of Subwavelength Nanostructures for Applications in Optics. Nano Lett. 2017, 17, 2973-2978.

(34) Wei, S.; Tian, F.; Ge, F.; Wang, X.; Zhang, G.; Lu, H.; Yin, J.; Wu, Z.; Qiu, L. Helical Nanofibrils of Block Copolymer for High-Performance Ammonia Sensors. ACS Appl. Mater. Interfaces 2018, 10, 22504-22512.

(35) Bas, S. Z.; Cummins, C.; Selkirk, A.; Borah, D.; Ozmen, M.; Morris, M. A. A Novel Electrochemical Sensor Based on Metal Ion Infiltrated Block Copolymer Thin Films for Sensitive and Selective Determination of Dopamine. ACS Appl. Nano Mater. 2019, 2, 7311-7318. 
(36) Bas, S. Z.; Cummins, C.; Borah, D.; Ozmen, M.; Morris, M. A. Electrochemical Sensing of Hydrogen Peroxide Using Block Copolymer Templated Iron Oxide Nanopatterns. Anal. Chem. 2018, 90, 1122-1128.

(37) Zou, Y.; Zhou, X.; Zhu, Y.; Cheng, X.; Zhao, D.; Deng, Y. sp²-Hybridized CarbonContaining Block Copolymer Templated Synthesis of Mesoporous Semiconducting Metal Oxides with Excellent Gas Sensing Property. Acc. Chem. Res. 2019, 52, 714-725.

(38) Nagpal, U.; Müller, M.; Nealey, P. F.; de Pablo, J. J. Free Energy of Defects in Ordered Assemblies of Block Copolymer Domains. ACS Macro Lett. 2012, 1, 418-422.

(39) Segal-Peretz, T.; Ren, J.; Xiong, S.; Khaira, G.; Bowen, A.; Ocola, L. E.; Divan, R.; Doxastakis, M.; Ferrier, N. J.; de Pablo, J.; Nealey, P. F. Quantitative Three-Dimensional Characterization of Block Copolymer Directed Self-Assembly on Combined Chemical and Topographical Prepatterned Templates. ACS Nano 2017, 11, 1307-1319.

(40) Li, W.; Müller, M. Thermodynamics and Kinetics of Defect Motion and Annihilation in the Self-Assembly of Lamellar Diblock Copolymers. Macromolecules 2016, 49, 6126-6138.

(41) Hur, S.-M.; Thapar, V.; Ramírez-Hernández, A.; Nealey, P. F.; de Pablo, J. J. Defect Annihilation Pathways in Directed Assembly of Lamellar Block Copolymer Thin Films. ACS Nano 2018, 12, 9974-9981.

(42) Doise, J.; Koh, J. H.; Kim, J. Y.; Zhu, Q.; Kinoshita, N.; Suh, H. S.; Delgadillo, P. R.; Vandenberghe, G.; Willson, C. G.; Ellison, C. J. Strategies for Increasing the Rate of Defect Annihilation in the Directed Self-Assembly of High- $\chi$ Block Copolymers. ACS Appl. Mater. Interfaces 2019, 11, 48419-48427.

(43) International Technology Roadmap for Semiconductors, 2011th ed.; Semiconductor Industry Association: San Jose, CA, 2011. https://www.semiconductors.org/resources/2011international-technology-roadmap-for-semiconductors-itrs/

(44) Hur, S.-M.; Khaira, G. S.; Ramírez-Hernández, A.; Müller, M.; Nealey, P. F.; de Pablo, J. J. Simulation of Defect Reduction in Block Copolymer Thin Films by Solvent Annealing. $A C S$ Macro Lett. 2015, 4, 11-15. 
(45) Gu, X.; Gunkel, I.; Hexemer, A.; Gu, W.; Russell, T. P. An In Situ Grazing Incidence XRay Scattering Study of Block Copolymer Thin Films During Solvent Vapor Annealing. Adv. Mater. 2014, 26, 273-281.

(46) Jin, C.; Olsen, B. C.; Luber, E. J.; Buriak, J. M. Nanopatterning via Solvent Vapor Annealing of Block Copolymer Thin Films. Chem. Mater. 2017, 29, 176-188.

(47) Selkirk, A.; Prochukhan, N.; Lundy, R.; Cummins, C.; Gatensby, R.; Kilbride, R.; Parnell, A.; Baez Vasquez, J.; Morris, M.; Mokarian-Tabari, P. Optimization and Control of Large Block Copolymer Self-Assembly via Precision Solvent Vapor Annealing. Macromolecules 2021.

(48) Lupi, F. F.; Giammaria, T. J.; Ceresoli, M.; Seguini, G.; Sparnacci, K.; Antonioli, D.; Gianotti, V.; Laus, M.; Perego, M. Rapid Thermal Processing of Self-Assembling Block Copolymer Thin Films. Nanotechnology 2013, 24, 315601.

(49) Williamson, L. D.; Seidel, R. N.; Chen, X.; Suh, H. S.; Rincon Delgadillo, P.; Gronheid, R.; Nealey, P. F. Three-Tone Chemical Patterns for Block Copolymer Directed Self-Assembly. ACS Appl. Mater. Interfaces 2016, 8, 2704-2712.

(50) Kim, S.-W.; Kim, E.; Lee, H.; Berry, B. C.; Kim, H.-C.; Ryu, D. Y. ThicknessDependent Ordering of Perpendicularly Oriented Lamellae in PS- $b$-PMMA Thin Films. Polymer 2015, 74, 63-69.

(51) Jin, C.; Murphy, J. N.; Harris, K. D.; Buriak, J. M. Deconvoluting the Mechanism of Microwave Annealing of Block Copolymer Thin Films. ACS Nano 2014, 8, 3979-3991.

(52) Borah, D.; Shaw, M. T.; Holmes, J. D.; Morris, M. A. Sub-10 nm Feature Size PS-bPDMS Block Copolymer Structures Fabricated by a Microwave-Assisted Solvothermal Process. ACS Appl. Mater. Interfaces 2013, 5, 2004-2012.

(53) Cummins, C.; Mokarian-Tabari, P.; Andreazza, P.; Sinturel, C.; Morris, M. A. Solvothermal Vapor Annealing of Lamellar Poly(styrene)-Block-Poly(d,l-Lactide) Block Copolymer Thin Films for Directed Self-Assembly Application. ACS Appl. Mater. Interfaces 2016, 8, 8295-8304.

(54) Majewski, P. W.; Yager, K. G. Millisecond Ordering of Block Copolymer Films via Photothermal Gradients. ACS Nano 2015, 9, 3896-3906. 
(55) Leniart, A. A.; Pula, P.; Sitkiewicz, A.; Majewski, P. W. Macroscopic Alignment of Block Copolymers on Silicon Substrates by Laser Annealing. ACS Nano 2020, 14, 4805-4815.

(56) Zhang, C.; Cavicchi, K. A.; Li, R.; Yager, K. G.; Fukuto, M.; Vogt, B. D. Thickness Limit for Alignment of Block Copolymer Films Using Solvent Vapor Annealing with Shear. Macromolecules 2018, 51, 4213-4219.

(57) Luo, M.; Scott, D. M.; Epps, T. H. Writing Highly Ordered Macroscopic Patterns in Cylindrical Block Polymer Thin Films via Raster Solvent Vapor Annealing and Soft Shear. ACS Macro Lett. 2015, 4, 516-520.

(58) Jo, S.; Jeon, S.; Kim, H.; Ryu, C. Y.; Lee, S.; Ryu, D. Y. Balanced Interfacial Interactions for Fluoroacrylic Block Copolymer Films and Fast Electric Field Directed Assembly. Chem. Mater. 2020, 32, 9633-9641.

(59) Gunkel, I. Directing Block Copolymer Self-Assembly on Patterned Substrates. Small 2018, 14, 1802872.

(60) Stoykovich, M. P.; Müller, M.; Kim, S. O.; Solak, H. H.; Edwards, E. W.; Pablo, J. J. de; Nealey, P. F. Directed Assembly of Block Copolymer Blends into Nonregular Device-Oriented Structures. Science 2005, 308, 1442-1446.

(61) Ober, C. K. A Dress Code for Block Copolymers. Nat. Nanotechnol. 2017, 12, 507-508.

(62) Suh, H. S.; Kim, D. H.; Moni, P.; Xiong, S.; Ocola, L. E.; Zaluzec, N. J.; Gleason, K. K.; Nealey, P. F. Sub-10-nm Patterning via Directed Self-Assembly of Block Copolymer Films with a Vapour-Phase Deposited Topcoat. Nat. Nanotechnol. 2017, 12, 575-581.

(63) Michman, E.; Langenberg, M.; Stenger, R.; Oded, M.; Schvartzman, M.; Müller, M.; Shenhar, R. Controlled Spacing Between Nanopatterned Regions in Block Copolymer Films Obtained by Utilizing Substrate Topography for Local Film Thickness Differentiation. ACS Appl. Mater. Interfaces 2019, 11, 35247-35254.

(64) Shin, J. Y.; Oh, Y. T.; Kim, S.; Lim, H. Y.; Lee, B.; Ko, Y. C.; Park, S.; Seon, S. W.; Lee, S. G.; Mun, S. S.; Kim, B. H. Hierarchical Self-Assembly of Thickness-Modulated Block Copolymer Thin Films for Controlling Nanodomain Orientations Inside Bare Silicon Trenches. Polymers 2021, 13, 553. 
(65) Ham, S.; Shin, C.; Kim, E.; Ryu, D. Y.; Jeong, U.; Russell, T. P.; Hawker, C. J. Microdomain Orientation of PS- $b$-PMMA by Controlled Interfacial Interactions. Macromolecules 2008, 41, 6431-6437.

(66) Smith, A. P.; Douglas, J. F.; Meredith, J. C.; Amis, E. J.; Karim, A. Combinatorial Study of Surface Pattern Formation in Thin Block Copolymer Films. Phys. Rev. Lett. 2001, 87, 015503.

(67) Kim, B. H.; Lee, H. M.; Lee, J.-H.; Son, S.-W.; Jeong, S.-J.; Lee, S.; Lee, D. I.; Kwak, S. U.; Jeong, H.; Shin, H.; Yoon, J.-B.; Lavrentovich, O. D.; Kim, S. O. Spontaneous Lamellar Alignment in Thickness-Modulated Block Copolymer Films. Adv. Funct. Mater. 2009, 19, 2584 2591.

(68) Black, C. T.; Forrey, C.; Yager, K. G. Thickness-Dependence of Block Copolymer Coarsening Kinetics. Soft Matter 2017, 13, 3275-3283.

(69) Olaya-Muñoz, D. A.; Nealey, P. F.; Hernández-Ortiz, J. P. Leveling of Polymer Grating Structures Upon Heating: Dimension Dependence on the Nanoscale and the Effect of Antiplasticizers. ACS Appl. Mater. Interfaces 2018, 10, 27432-27443.

(70) Ren, J.; Zhou, C.; Chen, X.; Dolejsi, M.; Craig, G. S. W.; Rincon Delgadillo, P. A.; Segal-Peretz, T.; Nealey, P. F. Engineering the Kinetics of Directed Self-Assembly of Block Copolymers Toward Fast and Defect-Free Assembly. ACS Appl. Mater. Interfaces 2018, 10, 23414-23423.

(71) Knoll, A.; Horvat, A.; Lyakhova, K. S.; Krausch, G.; Sevink, G. J. A.; Zvelindovsky, A. V.; Magerle, R. Phase Behavior in Thin Films of Cylinder-Forming Block Copolymers. Phys. Rev. Lett. 2002, 89, 035501.

(72) Sunday, D. F.; Dolejsi, M.; Chang, A. B.; Richter, L. J.; Li, R.; Kline, R. J.; Nealey, P. F.; Grubbs, R. H. Confinement and Processing Can Alter the Morphology and Periodicity of Bottlebrush Block Copolymers in Thin Films. ACS Nano 2020, 14, 17476-17486.

(73) Li, W.; Müller, M. Defects in the Self-Assembly of Block Copolymers and Their Relevance for Directed Self-Assembly. Annu. Rev. Chem. Biomol. Eng. 2015, 6, 187-216.

(74) Harrison, C.; Angelescu, D. E.; Trawick, M.; Cheng, Z.; Huse, D. A.; Chaikin, P. M.; Vega, D. A.; Sebastian, J. M.; Register, R. A.; Adamson, D. H. Pattern Coarsening in a 2D Hexagonal System. EPL 2004, 67, 800. 
(75) Vega, D. A.; Harrison, C. K.; Angelescu, D. E.; Trawick, M. L.; Huse, D. A.; Chaikin, P. M.; Register, R. A. Ordering Mechanisms in Two-Dimensional Sphere-Forming Block Copolymers. Phys. Rev. E 2005, 71, 061803.

(76) Stein, G. E.; Cochran, E. W.; Katsov, K.; Fredrickson, G. H.; Kramer, E. J.; Li, X.; Wang, J. Symmetry Breaking of In-Plane Order in Confined Copolymer Mesophases. Phys. Rev. Lett. 2007, 98, 158302.

(77) Tang, C.; Bang, J.; E. Stein, G.; Fredrickson, G. H.; Hawker, C. J.; Kramer, E. J.; Sprung, M.; Wang, J. Square Packing and Structural Arrangement of ABC Triblock Copolymer Spheres in Thin Films. Macromolecules 2008, 41, 4328-4339.

(78) Cao, B.; Adutwum, L. A.; Oliynyk, A. O.; Luber, E. J.; Olsen, B. C.; Mar, A.; Buriak, J. M. How To Optimize Materials and Devices via Design of Experiments and Machine Learning: Demonstration Using Organic Photovoltaics. ACS Nano 2018, 12, 7434-7444.

(79) Kirkey, A.; Luber, E. J.; Cao, B.; Olsen, B. C.; Buriak, J. M. Optimization of the Bulk Heterojunction of All-Small-Molecule Organic Photovoltaics Using Design of Experiment and Machine Learning Approaches. ACS Appl. Mater. Interfaces 2020, 12, 54596-54607.

(80) Nečas, D.; Klapetek, P. Gwyddion: An Open-Source Software for SPM Data Analysis. Open Phys. 2011, 10, 181-188.

(81) Otsu, N. A Threshold Selection Method from Gray-Level Histograms. IEEE Trans. Syst. Man Cybern. 1979, 9, 62-66.

(82) Pedregosa, F.; Varoquaux, G.; Gramfort, A.; Michel, V.; Thirion, B.; Grisel, O.; Blondel, M.; Prettenhofer, P.; Weiss, R.; Dubourg, V.; Vanderplas, J.; Passos, A.; Cournapeau, D.; Brucher, M.; Perrot, M.; Duchesnay, É. Scikit-Learn: Machine Learning in Python. J Mach Learn Res 2011, 12, 2825-2830.

(83) Jung, Y. S.; Ross, C. A. Orientation-Controlled Self-Assembled Nanolithography Using a Polystyrene-Polydimethylsiloxane Block Copolymer. Nano Lett. 2007, 7, 2046-2050.

(84) Jeong, J. W.; Park, W. I.; Kim, M.-J.; Ross, C. A.; Jung, Y. S. Highly Tunable SelfAssembled Nanostructures from a Poly(2-Vinylpyridine- $b$-Dimethylsiloxane) Block Copolymer. Nano Lett. 2011, 11, 4095-4101. 
(85) Leibler, L. Theory of Microphase Separation in Block Copolymers. Macromolecules 1980, 13, 1602-1617.

(86) Ohta, T.; Kawasaki, K. Equilibrium Morphology of Block Copolymer Melts. 1986, 19, 12.

(87) Bates, F. S.; Fredrickson, G. H. Block Copolymer Thermodynamics: Theory and Experiment. Annu. Rev. Phys. Chem. 1990, 41, 525-557.

(88) Jung, Y. S.; Ross, C. A. Solvent-Vapor-Induced Tunability of Self-Assembled Block Copolymer Patterns. Adv. Mater. 2009, 21, 2540-2545.

(89) Helfand, E.; Tagami, Y. Theory of the Interface Between Immiscible Polymers. II. $J$. Chem. Phys. 1972, 56, 3592-3601.

(90) Hashimoto, T.; Shibayama, M.; Kawai, H. Ordered Structure in Block Polymer Solutions. 4. Scaling Rules on Size of Fluctuations with Block Molecular Weight, Concentration, and Temperature in Segregation and Homogeneous Regimes. Macromolecules 1983, 16, 1093-1101.

(91) Cheng, L.-C.; Bai, W.; Martin, E. F.; Tu, K.-H.; Ntetsikas, K.; Liontos, G.;

Avgeropoulos, A.; Ross, C. A. Morphology, Directed Self-Assembly and Pattern Transfer from a High Molecular Weight Polystyrene-Block-Poly(dimethylsiloxane) Block Copolymer Film. Nanotechnology 2017, 28, 145301.

(92) Baruth, A.; Seo, M.; Lin, C. H.; Walster, K.; Shankar, A.; Hillmyer, M. A.; Leighton, C. Optimization of Long-Range Order in Solvent Vapor Annealed Poly(styrene)-Block-Poly(lactide) Thin Films for Nanolithography. ACS Appl. Mater. Interfaces 2014, 6, 13770-13781.

(93) Gasser, U.; Eisenmann, C.; Maret, G.; Keim, P. Melting of Crystals in Two Dimensions. ChemPhysChem 2010, 11, 963-970.

(94) Steinhardt, P. J.; Nelson, D. R.; Ronchetti, M. Bond-Orientational Order in Liquids and Glasses. Phys. Rev. B 1983, 28, 784-805.

(95) Euclidean Distance Mapping. Comput. Graph. Image Process. 1980, 14, 227-248.

(96) Han, E.; Stuen, K. O.; La, Y.-H.; Nealey, P. F.; Gopalan, P. Effect of Composition of Substrate-Modifying Random Copolymers on the Orientation of Symmetric and Asymmetric Diblock Copolymer Domains. Macromolecules 2008, 41, 9090-9097. 
(97) Albert, J. N. L.; Baney, M. J.; Stafford, C. M.; Kelly, J. Y.; Epps, T. H. Generation of Monolayer Gradients in Surface Energy and Surface Chemistry for Block Copolymer Thin Film Studies. ACS Nano 2009, 3, 3977-3986.

(98) Smith, A. P.; Douglas, J. F.; Amis, E. J.; Karim, A. Effect of Temperature on the Morphology and Kinetics of Surface Pattern Formation in Thin Block Copolymer Films. Langmuir 2007, 23, 12380-12387.

(99) Suh, H. S.; Kang, H.; Nealey, P. F.; Char, K. Thickness Dependence of Neutral Parameter Windows for Perpendicularly Oriented Block Copolymer Thin Films. Macromolecules 2010, 43, 4744-4751.

(100) Surowiec, I.; Vikström, L.; Hector, G.; Johansson, E.; Vikström, C.; Trygg, J. Generalized Subset Designs in Analytical Chemistry. Anal. Chem. 2017, 89, 6491-6497. 